\title{
Optimal Overhead Selection for Interference Alignment in Time-varying Two-user MIMO X Channel
}

\author{
Sudheesh P. G. ${ }^{*}$, Maurizio Magarini ${ }^{\dagger}$, P. Muthuchidambaranathan* \\ *Department of Electronics and Communication Engineering \\ National Institute of Technology, 620015 Tiruchirappalli, India \\ Email:pgsudheesh@gmail.com, muthuc@nitt.edu \\ ${ }^{\dagger}$ Dipartimento di Elettronica, Informazione e Bioingegneria \\ Politecnico di Milano, 20133 Milano, Italy \\ Email: maurizio.magarini@polimi.it
}

\begin{abstract}
Channel state information (CSI) at the transmitter and receiver is an essential requirement for interference alignment (IA) schemes. For moving users the channel coefficients vary with time and, therefore, it is required to update CSI both at the transmitter and receiver at regular intervals. Meanwhile it is important to note that frequent updates of CSI will reduce data rate and delayed updates will cause a large variation in CSI. In this context we explore the error performance of IA in two-user multiple-input multiple-output (MIMO) $X$ channel where the channel suffers continuous time-varying fading. The bit error rate (BER) performance of MIMO two-user $X$ channel is evaluated for different Doppler frequencies. We also propose a method for calculating optimal pilot overhead for time-varying channels by setting an upper bound on BER.
\end{abstract}

\section{INTRODUCTION}

The present day mobile devices like smart phones and hand held computers demand huge throughput, especially for multimedia traffic and video telephony. Dense deployment of transmitter base stations (BSs) increases the capacity of cellular networks to many folds. The dense deployment of transmitters leads to an inevitable product called interference and this uncontrolled interference limits the achievable gain in cellular networks. An interference channel reveals the communication scenario of transmitter-receiver pairs in which each transmitter sends information for its intended receiver and, at the same time, creates interference to the other receivers [1][4].

It is in this scenario that $\mathrm{X}$ network plays an important role. In the $\mathrm{X}$ network, each transmitter has separate message for each of the receivers [1], [2]. An X network with two transmitters and receivers is called an $\mathrm{X}$ channel. Multipleinput multiple-output (MIMO) allows us to increase data rate and/or to have more reliable wireless communication. Along with MIMO, the $\mathrm{X}$ network enables the users at the edge of the cell to be served by two or more BSs.

Among numerous interference mitigation techniques, interference alignment (IA) is a technique where interfering signals at each receiving node are confined into a subspace which does not contain that spanned by the signal of interest. The desired signal can therefore be recovered free from interference [1], [3], [5]. The fundamental concepts related to IA were introduced in [3], with emphasis on the temporal domain where a joint design of precoding matrices over multiple symbol extensions of the time-varying channels is proposed. To achieve perfect IA the availability of channel state information (CSI) is required both at the transmitters and at the receivers [6].

Since perfect CSI knowledge is available only in theory, in practical cases we must resort to the use of imperfect CSI obtained by means of channel estimation algorithms. In [7] the effect on bit error rate (BER) of imperfect channel knowledge on MIMO X-channel is evaluated. Estimation algorithms are therefore required [12] that involve an exchange of information among transmitting and receiving nodes. This inevitably implies an increase of the associated overhead with a consequent decrease on the overall information rate [8]-[10]. Studies were conducted for IA in block fading channel [11] and continuous fading channel [8], [10]. The optimum pilot overhead and IA update interval for $K$-user interference channel in continuous fading have been obtained analytically in [8].

In our work, we propose a method to optimize the pilot spacing and the feedback overhead to achieve IA in a twouser X network. Throughout the work, we assume continuous fading (symbol-by-symbol) where CSI is obtained in the timeslots allocated for pilots. Unlike [8], which uses analytical methods for $K$-user channel case, we perform Monte-Carlo simulations for finding the optimal overhead. The precoding at the transmitter is carried out using the latest available CSI. At the receiving side a linear interpolation scheme similar to that proposed in [13], [14] is carried out to have an estimate of the channel outside the time slots allocated for pilot overhead. Along with the optimal overhead selection, we also study the BER performance under different Doppler frequencies. In addition, the effect of the number of antennas on the BER performance is analyzed.

The paper is organized as follows. A review of IA for X channel is provided in Section II. In Section III the proposed scheme for finding optimal overhead fraction for IA under continuous fading for two-user X network is discussed. Section IV is devoted to numerical results while conclusions are drawn in Section V. 


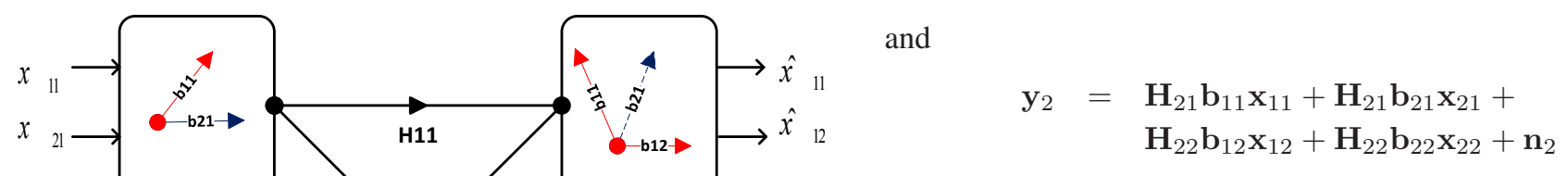

To satisfy the IA condition the interfering signals must span the same subspace. For aligning interference in receiver 1 we must satisfy the following conditions

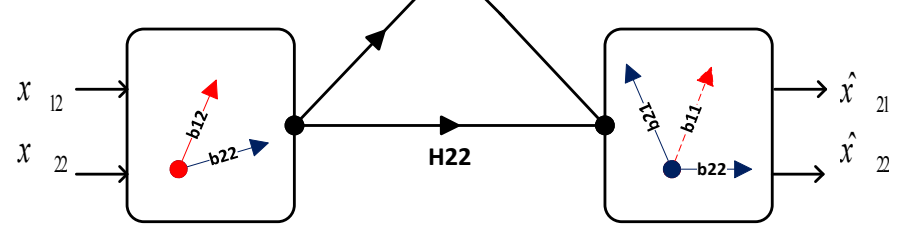

Fig. 1. Two-user MIMO X network.

\section{Notation}

The following notation is used in the paper: $(.)^{-1}$ is used for inverse of matrix . Vectors and matrices are represented by lower case and upper case boldface letters, respectively.

\section{INTERFERENCE ALIGNMENT IN X CHANNEL}

The diagram of the considered two-user MIMO X channel is shown in Figure 1. We consider the case with same number of antennas $A$ at the transmitter and the receiver. The $A \times 1$ received signal vectors for user 1 and user 2 are, respectively,

$$
\mathbf{y}_{1}=\mathbf{H}_{11} \mathbf{x}_{1}+\mathbf{H}_{12} \mathbf{x}_{2}+\mathbf{n}_{1}
$$

and

$$
\mathbf{y}_{2}=\mathbf{H}_{21} \mathbf{x}_{1}+\mathbf{H}_{22} \mathbf{x}_{2}+\mathbf{n}_{2},
$$

where $\mathbf{x}_{i}$ is the $A \times 1$ signal vector transmitted by the $i$-th user, $\mathbf{H}_{j i}$ is an $A \times A$ channel matrix between transmitter $i$ and receiver $j$, with $i, j \in\{1,2\}$, whose elements are assumed to be independent and identically distributed (i.i.d) complex Gaussian random variables with zero mean and unit variance, and $\mathbf{n}_{j}$ is an $A \times 1$ vector of i.i.d. complex Gaussian random variables with zero mean and variance $\sigma_{n}^{2}$. Each transmitter is intended to transmit independent messages to each of the receivers separately.

It is shown in [2] that the achievable degree of freedom (DoF) for an $\mathrm{X}$-channel is between the lowerbound $\frac{M N A}{M+N-1 / A}$ and the upperbound $\frac{M N A}{M+N-1}$. For two-user $\mathrm{X}$ channel we choose $A=3$ for achieving the desired DoF.

The two transmitted vectors are given by

$$
\mathbf{x}_{1}=\mathbf{b}_{11} \mathbf{x}_{11}+\mathbf{b}_{21} \mathbf{x}_{21}
$$

and

$$
\mathbf{x}_{2}=\mathbf{b}_{12} \mathbf{x}_{12}+\mathbf{b}_{22} \mathbf{x}_{22}
$$

where $x_{j i}$ is the message to be transmitted from transmitter $i$ to receiver $j$ and $\mathbf{b}_{j i}$ is the beamforming vector associated with $x_{j i}$. By substituting (3) and (4) in (1) and (2) respectively, we have

$$
\begin{aligned}
\mathbf{y}_{1}= & \mathbf{H}_{11} \mathbf{b}_{11} \mathbf{x}_{11}+\mathbf{H}_{11} \mathbf{b}_{21} \mathbf{x}_{21}+ \\
& \mathbf{H}_{12} \mathbf{b}_{12} \mathbf{x}_{12}+\mathbf{H}_{12} \mathbf{b}_{22} \mathbf{x}_{22}+\mathbf{n}_{1}
\end{aligned}
$$

$$
\begin{aligned}
& S P A N\left\{\mathbf{H}_{11} \mathbf{b}_{21}\right\}=S P A N\left\{\mathbf{H}_{12} \mathbf{b}_{22}\right\} \\
& S P A N\left\{\mathbf{H}_{22} \mathbf{b}_{12}\right\}=S P A N\left\{\mathbf{H}_{21} \mathbf{b}_{11}\right\}
\end{aligned}
$$

and

From the two above equations we have that a possible choice of beamforming vectors to align interference at the receivers is [2]

$$
\mathbf{b}_{22}=\mathbf{H}_{12}^{-1} \mathbf{H}_{11} \mathbf{b}_{21}
$$

and

$$
\mathbf{b}_{12}=\mathbf{H}_{22}^{-1} \mathbf{H}_{21} \mathbf{b}_{11} .
$$

Equations (9) and (10) define the original IA solution proposed in [5]. From the two equations it clearly appears that knowledge of CSI from other transmitter to the desired receiver is required at each transmitting node.

\section{OPTIMAL OVERHEAD SELECTION FOR X CHANNEL IA}

\section{A. Pilot overhead model for time-varying channel}

Figure 2 shows the pilot overhead model adopted in this paper. Each frame consists of $T$ time slots and the first slot in each frame is dedicated for CSI updating. The beamforming vectors at the transmitters and the matrices used to implement zero forcing IA decoding at the receivers are computed from the acquired CSI. We consider a continuous fading approach for transmission, which essentially means that the channel is varying in each time slot. The received signal at $k$-th time slot of frame $j$ is represented by

$$
\mathbf{y}_{1}^{j, k}=\mathbf{H}_{11}^{j, k} \mathbf{x}_{1}^{j, k}+\mathbf{H}_{12}^{j, k} \mathbf{x}_{2}^{j, k}+\mathbf{n}_{1}^{j, k}
$$

and

$$
\mathbf{y}_{2}^{j, k}=\mathbf{H}_{21}^{j, k} \mathbf{x}_{1}^{j, k}+\mathbf{H}_{22}^{j, k} \mathbf{x}_{2}^{j, k}+\mathbf{n}_{2}^{j, k},
$$

where the transmitted symbols at transmitter 1 and transmitter 2 are given by

$$
\mathbf{x}_{1}^{j, k}=\mathbf{b}_{11}^{j, k} \mathbf{x}_{11}^{j, k}+\mathbf{b}_{21}^{j, k} \mathbf{x}_{21}^{j, k}
$$

and

$$
\mathbf{x}_{2}^{j, k}=\mathbf{b}_{12}^{j, k} \mathbf{x}_{12}^{j, k}+\mathbf{b}_{22}^{j, k} \mathbf{x}_{22}^{j, k}
$$

respectively.

\section{B. Precoding and zero forcing decoding}

For achieving IA we perform precoding at transmitter and zero forcing decoding at the receiver. Channel knowledge is required to compute the precoder beamforming vectors and the zero forcing decoding matrices. The first slot in each frame is allotted for performing the CSI acquisition. The acquired CSI from each frame is represented as $\left[\mathbf{H}^{1}, \mathbf{H}^{2}, \mathbf{H}^{3}, \cdots\right]$. By this way CSI is made instantaneously available at the transmitter at the first slot of each frame. Precoding vectors for rest of the time slots in each frame are designed using CSI that is 


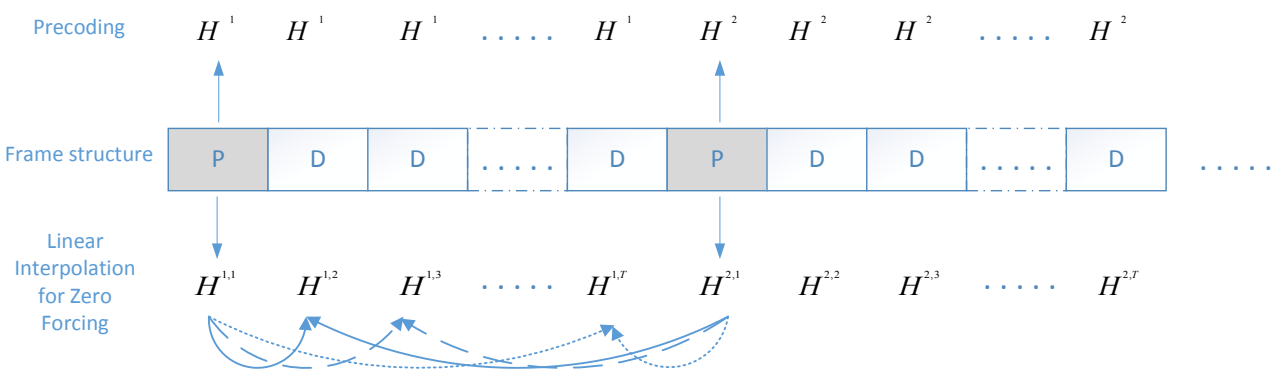

Fig. 2. Structure of the frame for channel estimation: $P$ are the pilot symbols and $D$ are the data.

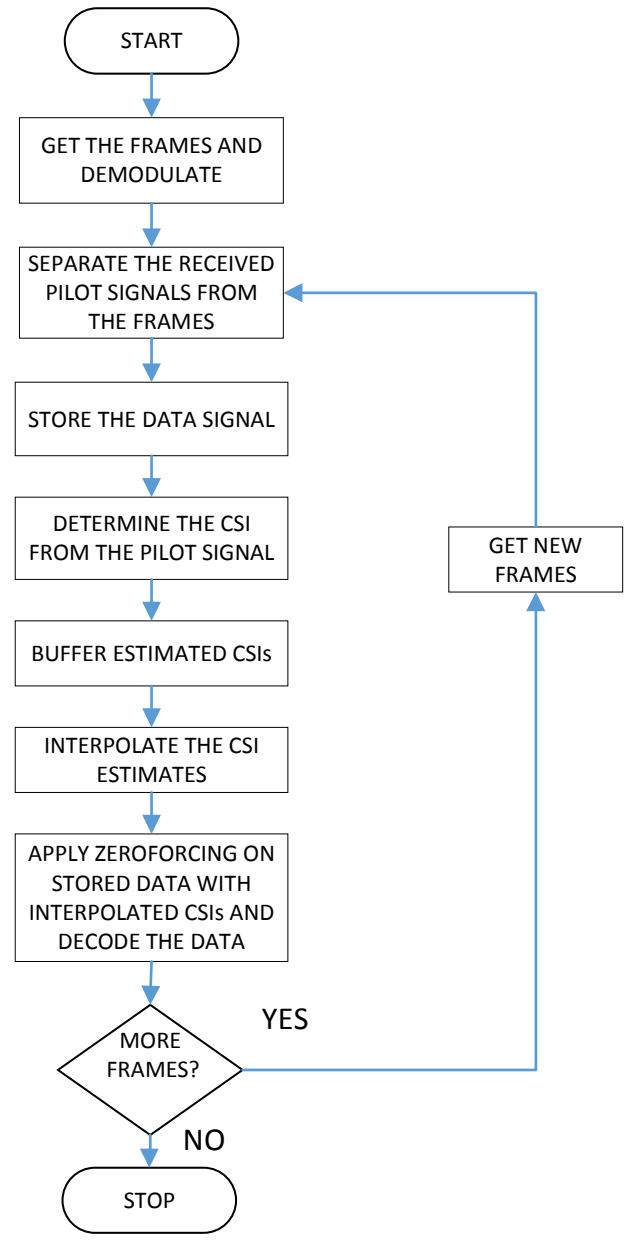

Fig. 3. Zero forcing using linearly interpolated channel estimates.

available at the beginning of the frame. For the zero forcing decoding matrices we consider that an estimated channel matrix is stored from at least one prior frame as described in [13] and [14]. At the $k$-th symbol of frame $j$, the estimated channel gain $\hat{\mathbf{H}}^{j, k}$ is calculated by linear interpolation of the channel estimates obtained from the previous and the upcoming pilot symbols, i.e., $\mathbf{H}^{j}$ and $\mathbf{H}^{j+1}$. The precoding and zero forcing decoding scheme implemented in the receiver of our proposed scheme is represented in Figure 3. Unlike [14], our algorithm uses zero forcing decoding matrices obtained by linearly interpolating the estimated channel matrices.

\section{Overhead optimization}

Increasing the overhead fraction decreases the data rate whereas decreasing the pilot overhead fraction leads to higher CSI imperfections thus making impossible the implementation of a practical solution for IA. Hence, the optimization of the pilot overhead to achieve the lowest possible BER and maximum throughput must be investigated.

It is worth noting that precoding is done by the best known CSI, which is acquired from the first slot of that frame. After setting a frame length we perform Monte Carlo simulation to obtain BER performance for different SNR values. In the Monte Carlo simulation different Doppler frequencies are used and corresponding BER performance of IA for the two-user X channel is obtained.

The key to find optimal overhead is to find maximum possible frame length for a particular BER threshold. In order to perform overhead optimization the following steps are followed:

1) Fix the SNR and perform Monte Carlo simulation to get the plot of the BER versus frame length for different Doppler frequencies.

2) Set the upper limit of BER.

3) Identify the maximum possible frame length which satisfies step 2.

4) With the fixed SNR, calculate the optimal overhead for the maximum possible length of the frame length.

5) Repeat the steps 1-4 with another SNR.

\section{NUMERICAL RESULTS}

In this Section, we evaluate the BER performance of IA for a two-user X-channel obtained by Monte Carlo simulation. In the first phase Monte Carlo simulations are performed to derive the BER performance versus SNR for a fixed length of the frame. In the second phase we perform simulations to study BER performance with respect to the pilot length for a fixed SNR.

A number of $10^{3}$ frames each of length 10 is generated. The first time slot in each frame contains the pilot symbol. The symbols $\mathbf{x}_{11}^{j, k}, \mathbf{x}_{12}^{j, k}, \mathbf{x}_{21}^{j, k}$ and $\mathbf{x}_{22}^{j, k}$ are chosen from a quadrature phase-shift keying (QPSK) constellation. The SNR is given by $\mathrm{SNR}=\frac{P_{a v g}}{\sigma_{n}^{2}}$, where $P_{a v g}$ is the average transmitted power from a single antenna. We assume a continuous fading Rayleigh channel where coefficients vary from symbolto-symbol. The channel at the $k$-th symbol of frame $j$ is represented by $\mathbf{H}^{j, k}$. 


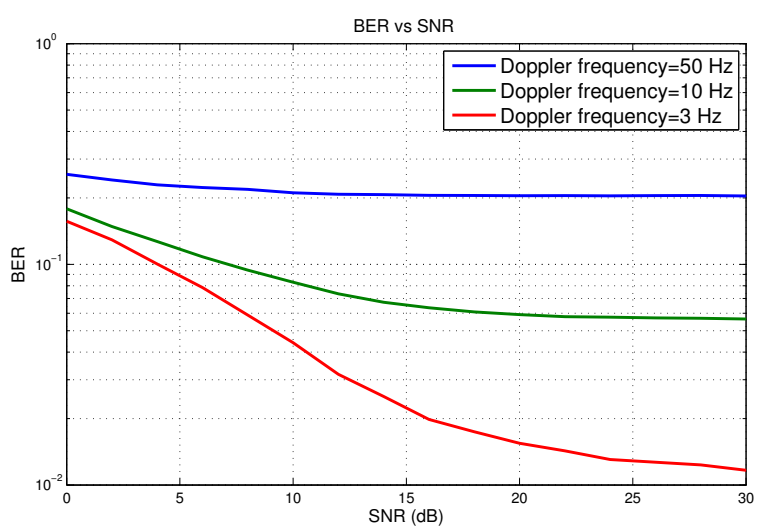

Fig. 4. BER versus SNR for different values of the Doppler frequency.

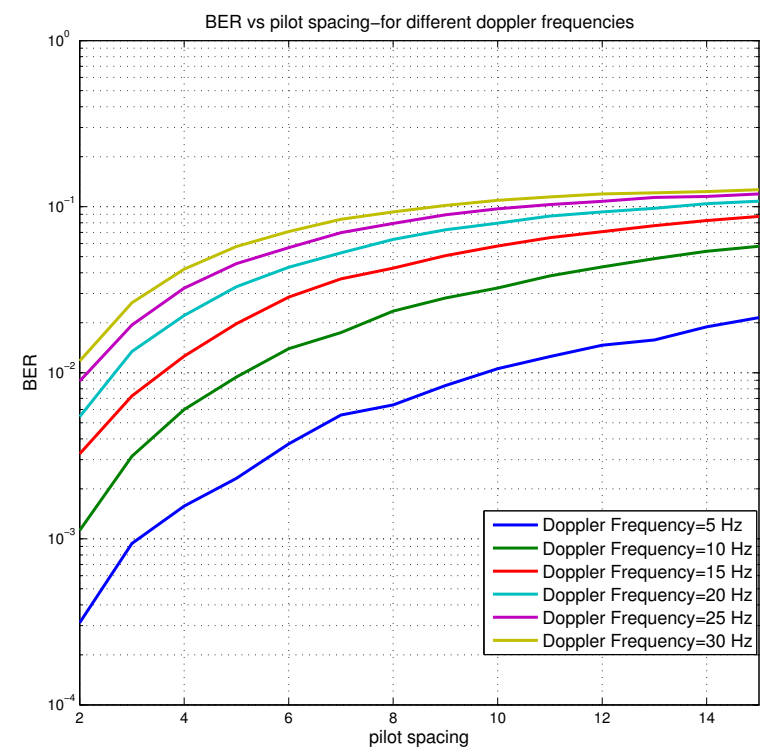

Fig. 5. BER versus frame length.

Figure 4 reports BER versus SNR at different Doppler frequencies. From our system model, it is considered that channel variation happens in each slot. The amount of channel variation is specified by Doppler frequency. The maximum Doppler frequency of $f_{m}=50 \mathrm{~Hz}$ corresponds to a vehicular velocity of $36 \mathrm{Km} / \mathrm{h}$ at $1.5 \mathrm{GHz}$. As in [6], each node is assumed to have 3 antennas, ie $A=3$. As one would expect BER decreases as SNR increases. The increasing Doppler frequency increases BER as the channel variation within the frame is higher. It is worth noting that the BER reaches an error floor as SNR increases. It is obvious that larger CSI imperfections used in the computation of precoding vectors and zero forcing matrices at higher Doppler frequencies causes this effect.

The computation of the optimal overhead is now considered. We choose $A=6$, and the reason for choosing such value of $A$ is discussed in Figure 6. The SNR is fixed to $40 \mathrm{~dB}$ and the frame length is varied from 2 to 15 . As in the first phase QPSK is considered. Obtained results are shown in Fig. 5.

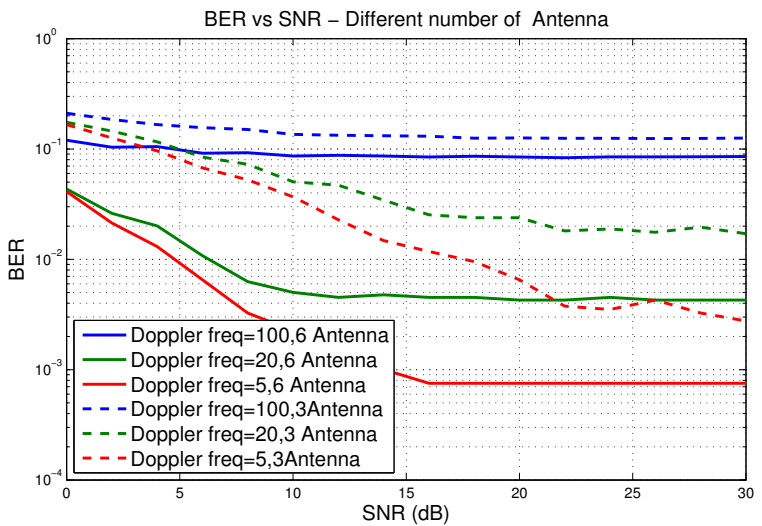

Fig. 6. Effect of number of antennas in IA

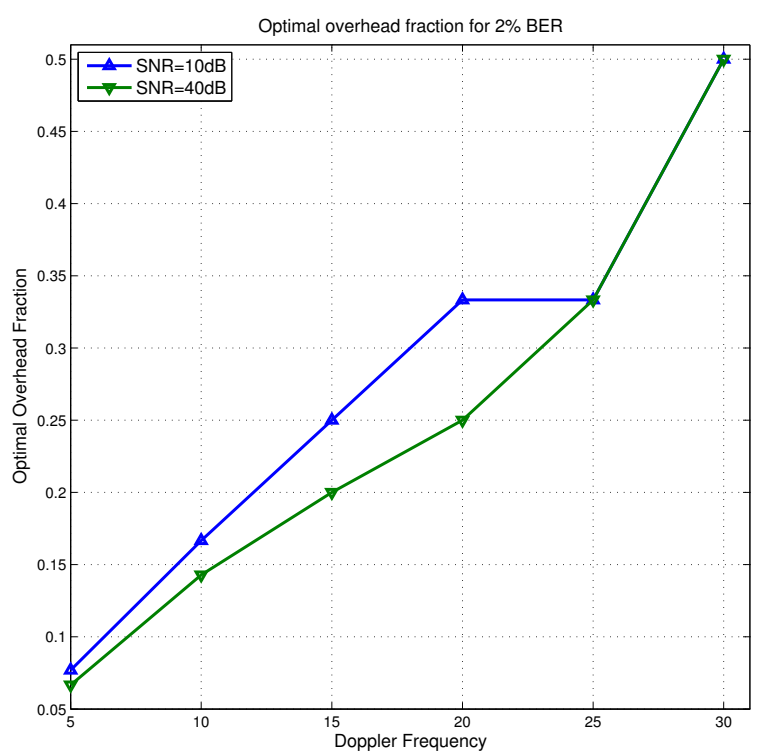

Fig. 7. Optimal overhead fraction for IA in a continuous fading Rayleigh channel.

Figure 6 reports the effect of number of antenna $A$ in BER performance. It is clear that BER performance improves as $A$ increases. A higher BER here is due to a frame length of 10 , which is the same in Figure 4 and Figure 5. A lesser frame length and doppler frequency offer improved BER as given in Figure 5. This is due to the fact that larger dimension for beamforming vector results in better correlation properties and, therefore, lesser BER. The number of antenna used in transmitter/ receiver decides the size of the channel matrix and hence the dimension of the precoding and zero-forcing vector. The correlation between these vectors plays crucial role in the performance of the system. Number of Antennas in two-user MIMO $X$ channel is taken as multiple of 3 , inorder to avoid non integer DoF.

The procedure for selecting optimal overhead is discussed in Sect. III-C. Figure 7 presents the optimal overhead fraction as a function of Doppler frequency. It is obvious that at higher Doppler frequencies, $50 \%$ pilot overhead is desirable 
to achieve IA. Since the frame length has to take an integer value, the optimal overhead must take values such as $33.33 \%$ and $50 \%$ at higher doppler frequencies. This is the reason for deviation from constant slope of the curve. At higher SNR, the CSI estimates are more accurate, leading to lesser BER. This implies that a constant BER would require lesser overhead for CSI estimation.

\section{CONCLUSION}

In the paper, the optimum pilot overhead and IA update interval is found by Monte Carlo simulations. Even though the optimal pilot overhead can be chosen for low values of the Doppler frequency, a pilot overhead of $50 \%$ is desirable at higher Doppler frequencies. Higher number of antennas at transmitter and receiver pull down the percentage of overhead.

We also present design of precoding at the transmitter and of zero forcing decoding at the receiver for continuous fading MIMO X channel with frame structure. Linear channel interpolation provides a less complex method to perform IA in a time-varying fading channel. The previous and the upcoming pilot symbols are used to find the CSI estimates.

\section{REFERENCES}

[1] M. A. Maddah-Ali, A. S. Motahari, and A. K. Khandani, "Communication over MIMO X channels: interference alignment, decomposition, and performance analysis," IEEE Trans. Inf. Theory, vol. 54, no. 8, pp. 3457-3470, Aug. 2008.

[2] S. A. Jafar and S. Shamai, "Degrees of freedom region of the MIMO X channel," IEEE Trans. Inf. Theory, vol. 54, no. 1, pp. 151-170, Jan. 2008.

[3] V. Cadambe and S. Jafar, "Interference alignment and degrees of freedom of the K-user interference channel," IEEE Trans. Inf. Theory, vol. 54, no. 8, pp. 3425-3441, Aug. 2008.
[4] C. Rose, S. Ulukus, and R. D. Yates, "Wireless systems and interference avoidance," IEEE Trans. Wireless Commun., vol. 1, no. 3, pp. 34573470, July 2002.

[5] S. A. Jafar, "Interference alignment: a new look at signal dimensions in a communication network," Foundations and Trends in Communication and Information Theory, vol. 7, no. 1, pp. 1-136, 2011.

[6] V. R. Cadambe, S. A. Jafar, "Interference alignment and the degrees of freedom of wireless X networks," IEEE Trans. Inf. Theory, vol. 55, no. 9, pp. 3893-3908, Sep. 2009.

[7] A. Mahmoud, M. El-Khamy, and K. Elsayed, "Interference alignment performance on MIMO X channels with imperfect channel knowledge," in Proc. Int. Workshop Signal Proc. Adv. Wireless Commun., pp. 239243, 2012.

[8] R. K. Mungara, G. George, A. Lozano, "Pilot-assisted interference alignment in time-selective fading channels," in Proc. GLOBECOM, pp. 3977-3982, Dec. 2013.

[9] H. Farhadi, M. N. Khormuji, M. Skoglund, "Pilot-assisted ergodic interference alignment for wireless networks," in Proc. Intern. Conf. Acoust., Speech, Signal Process., pp. 6186-6190, 2014.

[10] R. K. Mungara, G. George, and A. Lozano, "Overhead and spectral efficiency of pilot-assisted interference alignment in time-selective fading channels," IEEE Trans. Wireless Commun., vol. 13, no. 9, pp. 4884-4895, Sept. 2014.

[11] O. E. Ayach, A. Lozano, and R. W. Heath, Jr., "On the overhead of interference alignment: training, feedback, cooperation," IEEE Trans. Wireless Commun., vol. 11, no. 11, pp. 4192-4203, Nov. 2012

[12] B. Hassibi and B. M. Hochwald, "How much training is needed in multiple-antenna wireless links?" IEEE Trans. Inf. Theory, vol. 49, no. 4, pp. 951-963, Apr. 2003

[13] S. Savazzi and U. Spagnolini, "On the pilot spacing constraints for continuous time-varying fading channels," IEEE Trans. Commun., vol. 57, no. 11, pp. 3209-3213, Nov. 2009.

[14] R. F. Marchetto, T. A. Stewart, and P. K-M. Ho, "Compensation for multi-path interference using pilot symbols," U.S. Patent 5,414,734, issued May 9, 1995. 